\title{
Whole-Genome Sequences Analysis Displays Relationship of SARS-CoV-2 Delta Variant Between Four Local Cases and Passengers of a Flight from South Africa - Shenzhen City, Guangdong Province, China, June 2021
}

\author{
Yaqing $\mathrm{He}^{1,2}$; Renli Zhang ${ }^{1,2}$; Qiuying Lyu ${ }^{3}$; Bo Peng ${ }^{1,2}$; Shujiang Mei ${ }^{3}$; Ying Sun ${ }^{1,2}$; Dongfeng Kong ${ }^{3}$; Chaoqiong \\ Peng'; Ziquan Lyu'; Xinyi Wei ${ }^{1,2}$; Can Zhu ${ }^{1,2}$; Xiaoliang Xiao ${ }^{1,2}$; Shimin $\mathrm{Li}^{1,2}$; Qingju $\mathrm{Lu}^{1,2}$; Jiancheng Chen ${ }^{1,2}$; \\ Hang Zhang ${ }^{4}$; Xuan Zou ${ }^{4, * *}$; Tiejian Feng, ${ }^{2,4, *}$; Long Chen ${ }^{1,2, *}$
}

On June 14, 2021, a customs officer (Case A) went to the infirmary at Baoan International Airport in Shenzhen due to a runny nose and fever. He was admitted to the Central Hospital of Baoan immediately. This patient preliminarily tested positive for coronavirus disease 2019 (COVID-19) infection, caused by severe acute respiratory syndrome coronavirus 2 (SARS-CoV-2), using a quantitative realtime reverse transcription polymerase chain reaction (qRT-PCR) method in this hospital. Then, a mixed specimen of nasopharyngeal swab, oropharyngeal swab, and anal swab was sent to the virology laboratory of Shenzhen Center for Disease Control and Prevention (Shenzhen CDC) and was confirmed positive for SARS-CoV-2 by a qRT-PCR method simultaneously implemented in two commercial kits (Daan, Guangzhou, China and Bojie, Shanghai, China) (Supplementary Table S1, available in http://weekly. chinacdc.cn/). This patient tested negative for SARSCoV-2 on June 8, 2021 and participated in an epidemiological investigation and sampling in a flight from South Africa that arrived at Baoan International Airport on June 10, 2021. Between June 10, 2021 and June 25, 2021, a total of 39 passengers (Case 1 to 39) from this flight were confirmed to be infected with SARS-CoV-2 in the virology laboratory of Shenzhen CDC.

On June 17, 2021, a third-party laboratory detected SARS-CoV-2 in a mixed specimen of 10 swabs from ten individuals by qRT-PCR method, and the preliminary result was positive. This mixed specimen and one (Case B) of ten nasopharyngeal swabs from ten individuals were confirmed positive for SARSCoV-2 in the virology laboratory of Shenzhen CDC. Case B was a 22-year-old female who worked in a restaurant at Baoan International Airport. The third case (Case C) lived in Dongguan City and worked in
Nanshan District in Shenzhen City. He presented symptoms of chills, dry cough, diarrhea, and fever on June 12, 2021 and was confirmed to be infected with SARS-CoV-2 on June 18, 2021. The fourth case (Case D) lived and worked in Baoan District, Shenzhen. She was confirmed to be infected with SARS-CoV-2 during screening of key populations on June 20, 2021.

High-throughput sequencing was performed for 4 local SARS-CoV-2 strains and 39 imported SARSCoV-2 strains by Illumina Sequencing Technology. First, viral RNA was extracted directly from $200-\mu \mathrm{L}$ swab samples using a High Pure Viral RNA Kit (Roche, Germany). Second, viral RNA was reversetranscribed and amplified using ULSEN ${ }^{\circledR} 2019-\mathrm{nCoV}$ Whole Genome Capture Kit V-090418 (Beijing MicroFuture Technology Co., Ltd, Beijing, China). Third, the sequencing libraries were prepared using the Nextera ${ }^{\circledR}$ XT Library Prep Kit FC-131-1001(Illumina, Inc., San Diego, USA). The final viral-enriched libraries were sequenced using the MiSeq platform (Illumina, USA). The viral genome was assembled by MicronCoV $^{\circledR} \quad$ Analyzer M-881027 (Beijing MicroFuture Technology Co., Ltd, Beijing, China). Genome sequences of the 4 local SARS-CoV-2 strains and 28 of 39 imported SARS-CoV-2 strains were successfully determined. Virus strains from this study were genotyped using the online Pangolin COVID-19 Lineage Assigner (www.pangolin.cog-uk.io/). The evolutionary relationship of local SARS-CoV-2 strains and imported SARS-CoV-2 strains was inferred with the program MEGA version $\mathrm{X}$ (www.megasoft ware.net) (1). Nucleotide difference between viral genome sequences from this study and the reference sequence Wuhan-Hu-1 (GenBank no. NC_045512.2) was analyzed using the programs BioEdit 7.1.9 (www.bioedit.software.informer.com) and MEGA version $\mathrm{X}$. 
The 4 local SARS-CoV-2 strains and 26 of 28 imported SARS-CoV-2 strains from this study were assigned to lineage B.1.617.2 (Supplementary Table S2, available in http://weekly.chinacdc.cn/), which was the fourth variant of concern (VOC) (Delta variant). The other two imported SARS-CoV-2 strains were assigned to lineage B.1.351 and lineage C.1.2. Molecular phylogeny indicated that 30 SARS-CoV-2 Delta variants (lineage B.1.617.2) from this study formed 2 distinct clades (Figure 1). The imported strain hCoV-19/Shenzhen/IVDC-0610-01/2021 (Case1) clustered in clade $\mathrm{A}$ and showed the closest relationship to the Indian strain hCoV-19/India/GJNCDC-NIV-INSACOG-24095/2021 by an Audacity Instant search in Global initiative on sharing all influenza data (GISAID). The 4 local SARS-CoV-2 strains of lineage B.1.617.2 and 25 imported SARSCoV-2 strains of lineage B.1.617.2 clustered in clade B and showed the closest relationship or showed only one nucleotide difference to the British strain hCoV-

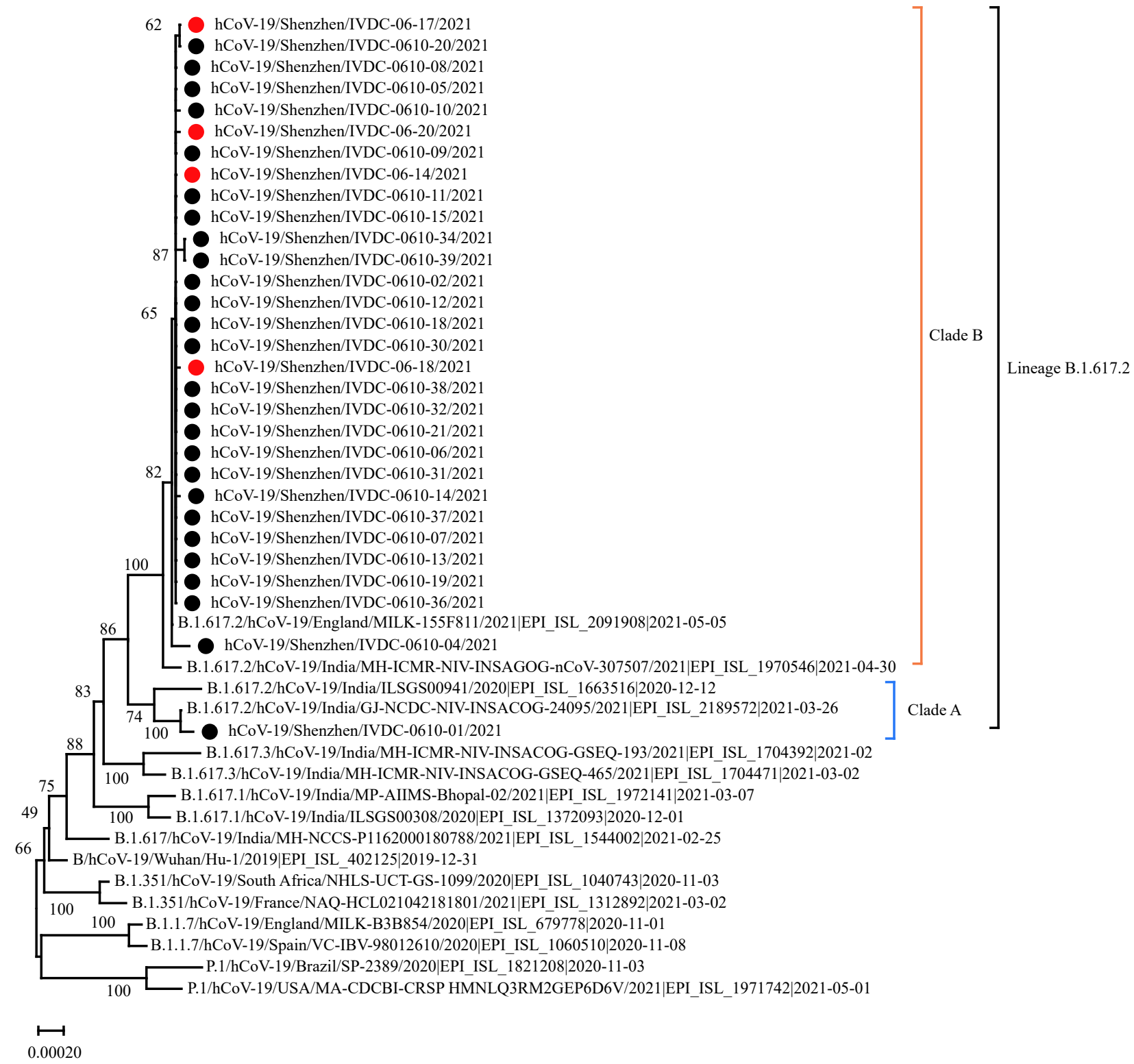

FIGURE 1. Evolutionary relationships of 30 SARS-CoV-2 strains of lineage B.1.617.2 from this study.

Note: Maximum likelihood phylogenetic tree was reconstructed for 30 SARS-CoV-2 strains from this study and 16 reference strains based on the whole-genome sequences. The best fit nucleotide substitution model used for phylogenetic reconstruction was TN93+G5+I. The scale bar represents a genetic distance of 0.0002 nucleotide substitutions per site. Bootstrap analysis (1,000 replicates) was used for statistical support of the tree. The 4 local strains were indicated by red dots, and 26 imported strains were indicated by black dots. 
TABLE 1. Nucleotide differences between 30 SARS-CoV-2 strains of lineage B.1.617.2 from this study and the reference strain Wuhan-Hu-1 (NC_045512.2).

\begin{tabular}{|c|c|c|c|}
\hline Clade & Case & Nucleotide variation & Nucleotide deletion mutation \\
\hline Clade A & Case 1 & $\begin{array}{l}\text { G210T, C241T, C799T, C3037T, G4181T, C6402T, } \\
\text { C7124T, C8986T, G9053T, C10029T, A11201G, } \\
\text { A11332G, G13812T, C14408T, G15451A, C16466T, } \\
\text { C19220T, C21618G, G21987A, T22917G, C22995A, } \\
\text { A23403G, C23604G, G24410A, C25413T, C25469T, } \\
\text { T26767C, T27638C, C27752T, C27874T, A28461G, } \\
\text { G28881T, G28916T, G29402T, and G29742T }\end{array}$ & $\begin{array}{l}\text { del22029-22034, } \\
\text { del28248-28253, } \\
\text { del28271 }\end{array}$ \\
\hline \multirow{8}{*}{ Clade B } & $\begin{array}{l}\text { Case A, Case 2, Case 5-9, } \\
\text { Case } 11-15, \text { Case 18, } \\
\text { Case } 19, \text { Case } 21, \\
\text { Case } 30-32, \text { Case } 36, \\
\text { Case } 38\end{array}$ & $\begin{array}{l}31 \text { variation sites: G210T, C241T, G410T, C3037T, } \\
\text { C5184T, A5584G, T9429C, C9891T, T11418C, } \\
\text { C11514T, C13019T, C14408T, G15451A, C16466T, } \\
\text { C21618G, G21987A, C22227T, T22917G, C22995A, } \\
\text { A23403G, C23604G, G24410A, C25469T, T26767C, } \\
\text { T27638C, A27677C, C27752T, A28461G, G28881T, } \\
\text { G29402T, and G29742T }\end{array}$ & $\begin{array}{l}\text { del510-518, del22029-22034, } \\
\text { del28248-28253, del28271 }\end{array}$ \\
\hline & Case B, Case 20 & 31 variation sites $+\mathrm{C} 21575 \mathrm{~T}$ & \multirow{3}{*}{$\begin{array}{l}\text { del510-518, del22029-22034, } \\
\text { del28248-28253, del28271 } \\
\text { del510-518, del22029-22034, } \\
\text { del28249, del28253, del28271 } \\
\text { del510-518, del22029-22034, } \\
\text { del28248-28253, del28271 }\end{array}$} \\
\hline & Case C & 31 variation sites $+\mathrm{C} 18431 \mathrm{~T}$ & \\
\hline & Case D & 31 variation sites $+C 10605 A$ & \\
\hline & Case 4 & $\begin{array}{l}31 \text { mutations - G210T - G410T - G21987A0 - } \\
\mathrm{C} 22227 \mathrm{~T}+\mathrm{G} 174 \mathrm{~T}+\mathrm{C} 1059 \mathrm{~T}+\mathrm{A} 5839 \mathrm{G}\end{array}$ & del28249, del28253, del28271 \\
\hline & Case 10 & 31 mutations $+\mathrm{C} 11665 \mathrm{~T}$ & \multirow{3}{*}{$\begin{array}{l}\text { del510-518, del22029-22034, } \\
\text { del28248-28253, del28271 } \\
\text { del510-518, del22029-22034, } \\
\text { del28248-28253, del28271 } \\
\text { del510-518, del22029-22034, } \\
\text { del28271 }\end{array}$} \\
\hline & Case 34, Case 39 & 31 mutations $+\mathrm{G} 3875 \mathrm{~A}+\mathrm{G} 6476 \mathrm{~T}$ & \\
\hline & Case 37 & 31 mutations $+\mathrm{A} 28249 \mathrm{~T}+\mathrm{C} 28253 \mathrm{~A}$ & \\
\hline
\end{tabular}

19/England/MILK-155F811/2021. These 29 SARS-

$\mathrm{CoV}-2$ strains of lineage B.1.617.2 showed at least 23 nucleotide differences to the strain hCoV-19/ Shenzhen/IVDC-0610-01/2021 (Case 1). In clade B, the imported strain hCoV-19/Shenzhen/IVDC-061004/2021 (Case 4) showed a certain degree of genetic distance to the other 28 virus strains from this study, which was also indicated by comparative analysis of genome sequences in Table 1. The local B.1.617.2 strain hCoV-19/Shenzhen/IVDC-06-14/2021 (Case A) showed $100 \%$ sequence identity to 19 imported B.1.617.2 strains (Table 1). These 20 SARS-CoV-2 strains of lineage B.1.617.2 shared 31 nucleotide variation sites and 22 deletion mutations compared with the reference strain Wuhan-Hu-1. Except for the variation sites above, an additional variation site was observed in genomes of the 3 local strains hCoV19/Shenzhen/IVDC-06-17/2021 (Case B), hCoV19/Shenzhen/IVDC-06-18/2021 (Case C), and hCoV19/Shenzhen/IVDC-06-20/2021 (Case D). The local strain hCoV-19/Shenzhen/IVDC-06-17/2021 (Case B) showed $100 \%$ sequence identity to the strain hCoV-19/Shenzhen/IVDC-0610-20/2021 from imported Case 20.

Among imported SARS-CoV-2 strains, genome sequences of 19 SARS-CoV-2 strains from imported passengers showed $100 \%$ sequence identity to each other, and these 19 passengers had no common exposure history before boarding. It suggested that at least one virus transmission occurred among the 19 passengers after boarding. The only strain from Case 1 belonged to clade A within lineage B.1.617.2, which indicated that Case 1 was not related to the infection in the cabin. Based on phylogenetic relationship and comparative analysis of genome sequences, we deduced that 3 virus strains from local cases (Cases B to D) and 5 virus strains from imported passengers (Cases 10, 20, 34,37 , and 39) were progeny viruses.

According to the report by $\mathrm{Li}$ et al (2), SARS-CoV-2 Delta variant strains from this study showed 18 to 28 nucleotide differences to those from the first outbreak of COVID-19 in Guangzhou, May 2021, which suggested that the SARS-CoV-2 Delta variant that caused local outbreaks of COVID-19 in Guangzhou and Shenzhen came from different sources. At present, the SARS-CoV-2 Delta variant is becoming the dominant variant worldwide and has been detected in at least 142 countries as of August 10, 2021. The SARS-CoV-2 Delta variant is posing new challenges on the control and prevention of COVID-19 due to its increased transmissibility compared with Alpha variant (lineage B.1.1.7) and capacity of immune escape (3-7).

In conclusion, whole-genome sequencing (WGS) confirmed that 4 local SARS-CoV-2 strains and 26 of 
28 imported SARS-CoV-2 strains from this study were Delta variants, and phylogenetic and comparative genome analyses showed close relationship between the four local SARS-CoV-2 strains of Delta variant and imported SARS-CoV-2 strains of Delta variant introduced from South Africa.

Acknowledgements: Baoan CDC, Nanshan CDC, and Shenzhen Third People's Hospital.

Conflicts of interest: No conflicts of interest.

Funding: Shenzhen Science and Technology Innovation Commission Key project (no. JSGG20200225152648408), the Shenzhen Science and Technology Innovation Commission COVID-19 Special Fund (no. JSGG20200207161926465), and Sanming Project of Medicine in Shenzhen (No.SZSM202011008).

doi: $10.46234 / \mathrm{ccdcw} 2021.215$

* Corresponding authors: Long Chen, chen_1_2011@163.com; Tiejian Feng, fengtiej@126.com; Xuan Zou, 914494557@qq.com.

\footnotetext{
${ }^{1}$ Institute of Pathogen Biology, Shenzhen Center for Disease Control and Prevention, Shenzhen, Guangdong, China; ${ }^{2}$ Shenzhen Center for Infectious Disease Control and Prevention, Chinese Academy of Medical Sciences, Shenzhen, Guangdong, China; ${ }^{3}$ Department of Communicable Diseases Control and Prevention, Shenzhen Center for Disease Control and Prevention, Shenzhen, Guangdong, China; ${ }^{4}$ Shenzhen Center for Disease Control and Prevention, Shenzhen, Guangdong, China; ${ }^{5}$ Laboratory of Molecular Epidemiology, Shenzhen Center for Disease Control and Prevention, Shenzhen, Guangdong, China.
}

Submitted: August 28, 2021; Accepted: September 29, 2021

\section{REFERENCES}

1. Kumar S, Stecher G, Li M, Knyaz C, Tamura K. MEGA X: molecular evolutionary genetics analysis across computing platforms. Mol Biol Evol 2018;35(6):1547 - 9. http://dx.doi.org/10.1093/molbev/msy096.

2. Li ZC, Nie K, Li KB, Hu Y, Song Y, Kang M, et al. Genome characterization of the first outbreak of COVID-19 delta variant B. 1. 617. 2 - Guangzhou City, Guangdong Province, China, May. China CDC Wkly 2021(27):587 - 9. http://dx.doi.org/10.46234/ccdcw2021. 151.

3. European Centre for Disease Prevention and Control. Implications for the EU/EEA on the spread of the SARSCoV-2 delta (B. 1.617. 2) variant of concern - 23 June 2021. ECDC: Stockholm. 2021. https://www. ecdc.europa.eu/sites/default/files/documents/Implications-for-the-EUEEA-on-the-spread-of-SARS-CoV-2-Delta-VOC-23-June-2021_2. pdf.[2021-08-14].

4. Campbell F, Archer B, Laurenson-Schafer H, Jinnai Y, Konings F, Batra $\mathrm{N}$, et al. Increased transmissibility and global spread of SARS-CoV-2 variants of concern as at June 2021. Euro Surveill 2021;26(24):2100509. http://dx.doi.org/10.2807/1560-7917.ES.2021.26.24.2100509.

5. Scientific Advisory Group for Emergencies. SPI-M-O: consensus statement on COVID-19, 3 June 2021. GOV. UK. 2021. https://www. gov.uk/government/publications/spi-m-o-consensus-statement-on-covid19-3-june-2021.[2021-08-14].

6. Planas D, Veyer D, Baidaliuk A, Staropoli I, Guivel-Benhassine F, Rajah $\mathrm{MM}$, et al. Reduced sensitivity of SARS-CoV-2 variant Delta to antibody neutralization. Nature 2021;596(7871):276 - 280. http://dx.doi.org/10. 1038/s41586-021-03777-9.

7. Liu C, Ginn HM, Dejnirattisai W, Supasa P, Wang BB, Tuekprakhon A, et al. Reduced neutralization of SARS-CoV-2 B. 1.617 by vaccine and convalescent serum. Cell 2021;184(16):4220 - 36.e13. http://dx. doi.org/10.1016/j.cell.2021.06.020. 
SUPPLEMENTARY TABLE S1. Demographic characteristics of 4 local cases and 39 imported cases, and specimen testing information.

\begin{tabular}{|c|c|c|c|c|c|}
\hline \multirow{2}{*}{ Case } & \multirow{2}{*}{ Gender } & \multirow{2}{*}{ Age (years) } & \multirow{2}{*}{$\begin{array}{c}\text { Date of first positive detection } \\
\text { of COVID-19 virus }\end{array}$} & \multicolumn{2}{|c|}{ Ct value (ORF1ab/N) of specimen used for sequencing } \\
\hline & & & & Daan & Bojie \\
\hline Case A & Male & 30 & June 14, 2021 & $14 / 18$ & $16 / 18$ \\
\hline Case B & Female & 21 & June 17, 2021 & $23 / 21$ & $22 / 22$ \\
\hline Case C & Male & 35 & June 18, 2021 & $24 / 22$ & $22 / 24$ \\
\hline Case D & Female & 64 & June 20, 2021 & $30 / 30$ & $35 / 28$ \\
\hline Case 1 & Male & 36 & June 11, 2021 & $31 / 31$ & $30 / 31$ \\
\hline Case 2 & Male & 35 & June 11, 2021 & $31 / 31$ & $26 / 28$ \\
\hline Case 3 & Female & 42 & June 11, 2021 & $33 / 34$ & $34 / 34$ \\
\hline Case 4 & Female & 58 & June 11, 2021 & $30 / 27$ & $27 / 28$ \\
\hline Case 5 & Male & 53 & June 13, 2021 & $17 / 16$ & $16 / 17$ \\
\hline Case 6 & Male & 32 & June 14, 2021 & $24 / 23$ & $24 / 23$ \\
\hline Case 7 & Male & 52 & June 14, 2021 & $17 / 17$ & $16 / 17$ \\
\hline Case 8 & Male & 33 & June 15, 2021 & $17 / 15$ & $15 / 17$ \\
\hline Case 9 & Male & 47 & June 15, 2021 & $16 / 16$ & $15 / 16$ \\
\hline Case 10 & Male & 64 & June 15, 2021 & $21 / 20$ & $20 / 21$ \\
\hline Case 11 & Male & 40 & June 15, 2021 & $15 / 14$ & $14 / 15$ \\
\hline Case 12 & Male & 50 & June 15, 2021 & $18 / 17$ & $16 / 18$ \\
\hline Case 13 & Male & 33 & June 15, 2021 & $28 / 26$ & $27 / 28$ \\
\hline Case 14 & Female & 31 & June 15, 2021 & $19 / 17$ & $18 / 17$ \\
\hline Case 15 & Male & 61 & June 15, 2021 & $14 / 14$ & $15 / 15$ \\
\hline Case 16 & Female & 38 & June 15, 2021 & $35 / 34$ & $36 / 33$ \\
\hline Case 17 & Male & 39 & June 15, 2021 & $37 / 40$ & Undet/35 \\
\hline Case 18 & Female & 50 & June 15,2021 & $14 / 15$ & $14 / 14$ \\
\hline Case 19 & Male & 35 & June 15, 2021 & $17 / 16$ & $16 / 17$ \\
\hline Case 20 & Male & 58 & June 15, 2021 & $16 / 15$ & $15 / 16$ \\
\hline Case 21 & Female & 47 & June 15, 2021 & $29 / 25$ & $26 / 26$ \\
\hline Case 22 & Male & 29 & June 15, 2021 & $35 / 35$ & 38/Undet \\
\hline Case 23 & Male & 32 & June 15, 2021 & $38 / 36$ & Undet/35 \\
\hline Case 24 & Male & 63 & June 15, 2021 & $35 / 34$ & $35 / 32$ \\
\hline Case 25 & Female & 63 & June 15, 2021 & $35 / 33$ & $33 / 33$ \\
\hline Case 26 & Male & 45 & June 15, 2021 & $38 / 35$ & Undet/Undet \\
\hline Case 27 & Male & 44 & June 15, 2021 & Undet/38 & Undet/35 \\
\hline Case 28 & Male & 61 & June 15, 2021 & Undet/37 & $37 /$ Undet \\
\hline Case 29 & Male & 58 & June 15, 2021 & 37/Undet & Undet/36 \\
\hline Case 30 & Male & 9 & June 15, 2021 & $21 / 21$ & $22 / 22$ \\
\hline Case 31 & Female & 45 & June 15, 2021 & $28 / 28$ & $27 / 28$ \\
\hline Case 32 & Male & 42 & June 15, 2021 & $15 / 15$ & $14 / 15$ \\
\hline Case 33 & Male & 35 & June 16, 2021 & $26 / 24$ & $23 / 26$ \\
\hline Case 34 & Male & 1 & June 16, 2021 & $30 / 27$ & $27 / 29$ \\
\hline Case 35 & Female & 24 & June 17, 2021 & $34 / 30$ & $32 / 30$ \\
\hline Case 36 & Male & 25 & June 17, 2021 & $19 / 18$ & $19 / 18$ \\
\hline Case 37 & Female & 32 & June 19, 2021 & $22 / 19$ & $19 / 20$ \\
\hline Case 38 & Male & 38 & June 19, 2021 & $31 / 29$ & $28 / 30$ \\
\hline Case 39 & Female & 28 & June 25, 2021 & $18 / 16$ & $17 / 18$ \\
\hline
\end{tabular}

Note: There were 43 subjects in total, and the male/female ratio and mean age of subjects was 2.58:1 and 40.9 years, respectively. Abbreviations: Undet=Undetected; Ct=Cycle threshold; COVID-19=Coronavirus disease 2019. 
SUPPLEMENTARY TABLE S2. Genome sequence information for 30 SARS-CoV-2 strains of lineage B.1.617.2 from this study.

\begin{tabular}{|c|c|c|c|}
\hline Case & Virus name & $\begin{array}{l}\text { Length in nt } \\
\text { (\% GC content) }\end{array}$ & $\begin{array}{c}\text { Closest strain by an audacity instant } \\
\text { search in GISAID }\end{array}$ \\
\hline Case A & hCoV-19/Shenzhen/IVDC-06-14/2021 & 29,813nt (37.97) & hCoV-19/England/MILK-155F811/2021 \\
\hline Case B & hCoV-19/Shenzhen/IVDC-06-17/2021 & 29,562nt (37.98) & hCoV-19/England/MILK-155F811/2021 \\
\hline Case C & hCoV-19/Shenzhen/IVDC-06-18/2021 & 29,428 nt (37.98) & hCoV-19/England/MILK-155F811/2021 \\
\hline Case D & hCoV-19/Shenzhen/IVDC-06-20/2021 & 29,612nt (37.98) & hCoV-19/England/MILK-155F811/2021 \\
\hline Case 1 & hCoV-19/Shenzhen/IVDC-0610-01/2021 & 29,702nt (37.96) & hCoV-19/India/GJ-NCDC-NIV-INSACOG-24095/2021 \\
\hline Case 2 & hCoV-19/Shenzhen/IVDC-0610-02/2021 & 29,813nt (37.97) & hCoV-19/England/MILK-155F811/2021 \\
\hline Case 4 & hCoV-19/Shenzhen/IVDC-0610-04/2021 & 29,836nt (37.96) & hCoV-19/England/MILK-155F811/2021 \\
\hline Case 5 & hCoV-19/Shenzhen/IVDC-0610-05/2021 & 29,555nt (37.99) & hCoV-19/England/MILK-155F811/2021 \\
\hline Case 6 & hCoV-19/Shenzhen/IVDC-0610-06/2021 & 29,657 nt $(38.00)$ & hCoV-19/England/MILK-155F811/2021 \\
\hline Case 7 & hCoV-19/Shenzhen/IVDC-0610-07/2021 & 29,814 nt $(37.97)$ & hCoV-19/England/MILK-155F811/2021 \\
\hline Case 8 & hCoV-19/Shenzhen/IVDC-0610-08/2021 & 29,824 nt $(37.97)$ & hCoV-19/England/MILK-155F811/2021 \\
\hline Case 9 & hCoV-19/Shenzhen/IVDC-0610-09/2021 & 29,831 nt (37.96) & hCoV-19/England/MILK-155F811/2021 \\
\hline Case 10 & hCoV-19/Shenzhen/IVDC-0610-10/2021 & 29,833nt (37.95) & hCoV-19/England/MILK-155F811/2021 \\
\hline Case 11 & hCoV-19/Shenzhen/IVDC-0610-11/2021 & 29,830nt (37.96) & hCoV-19/England/MILK-155F811/2021 \\
\hline Case 12 & hCoV-19/Shenzhen/IVDC-0610-12/2021 & 29,830nt (37.96) & hCoV-19/England/MILK-155F811/2021 \\
\hline Case 13 & hCoV-19/Shenzhen/IVDC-0610-13/2021 & 29,710nt (37.99) & hCoV-19/England/MILK-155F811/2021 \\
\hline Case 14 & hCoV-19/Shenzhen/IVDC-0610-14/2021 & $29,551 \mathrm{nt}(37.98)$ & hCoV-19/England/MILK-155F811/2021 \\
\hline Case 15 & hCoV-19/Shenzhen/IVDC-0610-15/2021 & 29,834nt (37.96) & hCoV-19/England/MILK-155F811/2021 \\
\hline Case 18 & hCoV-19/Shenzhen/IVDC-0610-18/2021 & 29,820 nt $(37.96)$ & hCoV-19/England/MILK-155F811/2021 \\
\hline Case 19 & hCoV-19/Shenzhen/IVDC-0610-19/2021 & 29,828 nt $(37.96)$ & hCoV-19/England/MILK-155F811/2021 \\
\hline Case 20 & hCoV-19/Shenzhen/IVDC-0610-20/2021 & 29,820nt (37.96) & hCoV-19/England/MILK-155F811/2021 \\
\hline Case 21 & hCoV-19/Shenzhen/IVDC-0610-21/2021 & 29,574nt (37.99) & hCoV-19/England/MILK-155F811/2021 \\
\hline Case 30 & hCoV-19/Shenzhen/IVDC-0610-30/2021 & $29,827 n t(37.97)$ & hCoV-19/England/MILK-155F811/2021 \\
\hline Case 31 & hCoV-19/Shenzhen/IVDC-0610-31/2021 & 29,829nt (37.96) & hCoV-19/England/MILK-155F811/2021 \\
\hline Case 32 & hCoV-19/Shenzhen/IVDC-0610-32/2021 & 29,849nt (37.96) & hCoV-19/England/MILK-155F811/2021 \\
\hline Case 34 & hCoV-19/Shenzhen/IVDC-0610-34/2021 & 29,809nt (37.97) & hCoV-19/England/MILK-155F811/2021 \\
\hline Case 36 & hCoV-19/Shenzhen/IVDC-0610-36/2021 & $29,819 n t(37.96)$ & hCoV-19/England/MILK-155F811/2021 \\
\hline Case 37 & hCoV-19/Shenzhen/IVDC-0610-37/2021 & 29,827nt (37.97) & hCoV-19/England/MILK-155F811/2021 \\
\hline Case 38 & hCoV-19/Shenzhen/IVDC-0610-38/2021 & 29,553nt (37.99) & hCoV-19/England/MILK-155F811/2021 \\
\hline Case 39 & hCoV-19/Shenzhen/IVDC-0610-39/2021 & 29,797nt (37.94) & hCoV-19/England/MILK-155F811/2021 \\
\hline
\end{tabular}

Abbreviation: $\mathrm{nt}=$ Nucleotide. 